\title{
EMBEDDING ORTHOGONAL PARTIAL LATIN SQUARES
}

\author{
CHARLES C. LINDNER
}

\begin{abstract}
Two partial latin squares are orthogonal provided that when they are superimposed any ordered pairs obtained are distinct. The purpose of this paper is to show that any collection of pairwise orthogonal finite partial latin squares can be embedded into pairwise orthogonal finite latin squares.
\end{abstract}

1. Introduction. In 1960 Trevor Evans [2] proved that a partial latin square of order $n$ can always be embedded in some latin square of order $t$ for every $t \geqslant 2 n$. In this same paper Evans raised the question as to whether a pair of finite partial latin squares which are orthogonal (when superimposed any ordered pairs obtained are distinct) can be embedded in a pair of orthogonal finite latin squares. The purpose of this paper is to answer this question affirmatively by giving a more general result. In particular, we prove that any collection of pairwise orthogonal partial latin squares can be embedded into pairwise orthogonal finite latin squares. To keep from using the word "finite" excessively, in what follows everything is finite regardless of whether or not it is mentioned.

2. Definitions. A (partial) latin square is an $n \times n$ array such that each of the integers $1,2, \ldots, n$ occurs (at most once) exactly once in each row and column. The (partial) latin square $P$ is embedded in the latin square $Q$ provided that the upper left-hand corner of $Q$ agrees with $P$. Two partial latin squares of the same size are orthogonal provided that when they are superimposed any ordered pairs obtained are distinct.

EXAMPLE. The three partial latin squares of order 4 given below are pairwise orthogonal. The 2 ordered pairs obtained by superimposing $A$ and $B$

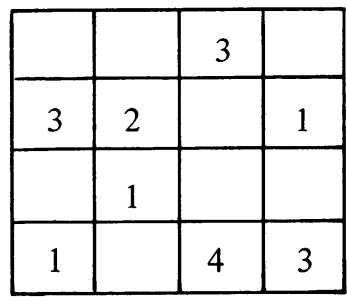

A

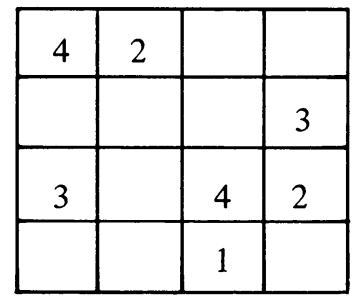

B

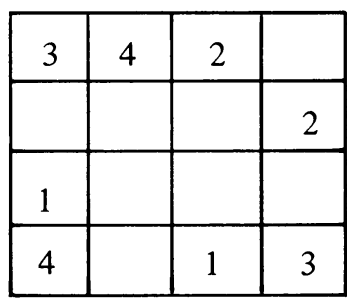

C

Presented to the Society, January 22, 1976; received by the editors August 29, 1975. AMS (MOS) subject classifications (1970). Primary 05B15.

Key words and phrases. Partial latin square, orthogonal latin squares, block designs. 
are $(1,3)$ and $(4,1)$; the 5 ordered pairs obtained by superimposing $A$ and $C$ are $(3,2),(1,2),(1,4),(4,3)$, and $(3,3)$; and the 5 ordered pairs obtained by superimposing $B$ and $C$ are $(4,3),(2,4),(3,2),(3,1)$, and $(1,1)$. In each case the ordered pairs are distinct.

By a (partial) $(2, k)$-Steiner system is meant a pair $(P, b)$ such that $P$ is a finite set and $b$ is a collection of $k$-element subsets of $P$ (called blocks) such that every pair of distinct elements of $P$ belonging to (at most) exactly one block of $b$. The (partial) $(2, k)$-Steiner system $(P, b)$ is said to be embedded in the $(2, k)$-Steiner system $(Q, q)$ provided that $P \subseteq Q$ and $b \subseteq q$. In [3], B. Ganter has shown that if $k-1$ is a power of a prime, then a finite partial $(2, k)$-Steiner system can be embedded in a finite $(2, k)$-Steiner system. R. Quackenbush obtained a similar (but not the same) result in [5] by proving that if $k$ is a power of a prime then a finite partial $(2, k)$-Steiner system can be embedded in a finite $(2, k)$-Steiner system. (Subsequently, Ganter has obtained a much more general embedding theorem along these lines [4].) With the above definitions in hand we proceed to the main result in this paper.

3. Embedding orthogonal partial latin squares. Let $P_{1}, P_{2}, \ldots, P_{t}$ be pairwise orthogonal partial latin squares of order $n$ based on $N=\{1,2, \ldots, n\}$. For each $i=1,2, \ldots, n$, let $A_{i}=\left\{a_{1 i}, a_{2 i}, \ldots, a_{n i}\right\}$ and $X_{i}$ a set of symbols disjoint from $A_{i}$ containing as many elements as there are empty cells in $P_{i}$. Take the sets $N, A_{1}, X_{1}, A_{2}, X_{2}, \ldots, A_{t}, X_{t}$ to be pairwise disjoint.

Finally, let $R=\left\{r_{1}, r_{2}, \ldots, r_{n}\right\}$ and $C=\left\{c_{1}, c_{2}, \ldots, c_{n}\right\}$ be disjoint sets each of which is disjoint from each of the above sets. Denote by $P$ the union of all of the above sets. Now replace each occurrence of $j$ in $P_{i}$ by $a_{j i}$ and fill in the empty cells with the symbols from $X_{i}$ so that each symbol in $X_{i}$ is used exactly once. Denote the resulting arrays by $P_{1}^{*}, P_{2}^{*}, \ldots, P_{t}^{*}$. Define a collection $b$ of $n^{2}(t+2)$-subsets of $P$ as follows: For each of the $n^{2}$ ordered pairs $(i, j) ; i, j \in\{1,2, \ldots, n\}$; set $b(i, j)=\left\{r_{i}, c_{j}, z_{1}, z_{2}, \ldots, z_{t}\right\}$ where $z_{i}$ is the entry in cell $(i, j)$ of $P_{i}^{*}$. Take $b=\{b(i, j) \mid$ all $i, j \in\{1,2, \ldots, n\}\}$. Claim: $(P, b)$ is a partial $(2, t+2)$-Steiner system. So let $b(i, j)=\left\{r_{i}, c_{j}, x_{1}, x_{2}, \ldots, x_{t}\right\}$ and $b(p, q)=\left\{r_{p}, c_{q}, y_{1}, y_{2}, \ldots, y_{t}\right\}$ be any two blocks in $b$. We show that $b(i, j)$ and $b(p, q)$ have at most one element in common. There are three cases to consider.

(1) $i=p$. In this case $b(i, j)$ and $b(p, q)$ have the element $r_{i}=r_{p}$ in common. Since $j \neq q, c_{j} \neq c_{q}$. Since $P_{i}^{*}$ and $P_{j}^{*}$ are based on disjoint sets of symbols we cannot have $x_{i}=y_{j}$ for $i \neq j$. If $x_{s}=y_{s}$ for some $s$, then cells $(i, j)$ and $(i$, $q), j \neq q$, are occupied by the same symbol in $P_{s}^{*}$ which, of course, cannot be.

(2) $j=q$. Analogous to case (1).

(3) $i \neq p$ and $j \neq q$. The only possibility here for $b(i, j)$ and $b(p, q)$ to have two elements in common is for $x_{u}=y_{u}$ and $x_{v}=y_{v}$ for some $u \neq v$. The only possibility for this to happen is that cells $(i, j)$ and $(p, q)$ were occupied in $P_{u}$ with the same symbol and also occupied by the same symbol in $P_{v}$. Since $P_{u}$ and $P_{v}$ are orthogonal this cannot happen. Combining all three cases shows that $(P, b)$ is a partial $(2, t+2)$-Steiner system. We can assume that $t+2$ is a power of a prime, and so by Quackenbush's theorem [5] embed $(P, b)$ in a finite $(2, t+2)$-Steiner system $(Q, q)$.

For each block $s \in q$ define any $t$ mutually orthogonal idempotent latin squares $s_{1}, s_{2}, \ldots, s_{t}$ based on $s$ with the following exception. If $s=b(i, j)$ 
$=\left\{r_{i}, c_{j}, x_{1}, x_{2}, \ldots, x_{t}\right\}$, take $s_{k}$ to be the latin square with cell $(i, j)$ occupied by the entry in cell $(i, j)$ of $P_{k}^{*}$. Construct for each $i=1,2, \ldots, t$ a latin square $Q_{i}$ from $(Q, q)$ by using for each block $s \in q$ the latin square $s_{i}$ defined above. This is the well-known Bose and Shrikhande construction [1] for mutually orthogonal latin squares and so the resulting latin squares $Q_{1}, Q_{2}$, $\ldots, Q_{t}$ are mutually orthogonal. If for each $i=1,2, \ldots, n$ we apply the permutation $\left(1 a_{1 i}\right)\left(2 a_{2 i}\right)\left(3 a_{3 i}\right), \ldots,\left(n a_{n i}\right)$ to the latin square $Q_{i}$ we obtain pairwise orthogonal latin squares $Q_{1}^{*}, Q_{2}^{*}, \ldots, Q_{t}^{*}$ with the property that if cell $(i, j)$ of $P_{m}$ is occupied by $k$, then in $Q_{m}^{*}$ cell $\left(r_{i}, c_{j}\right)$ is occupied by $k$. Hence if the permutation $\left(1 r_{1}\right)\left(2 r_{2}\right), \ldots,\left(n r_{n}\right)$ is applied to the rows of each $Q_{i}^{*}$ followed by the permutation $\left(1 c_{1}\right)\left(2 c_{2}\right), \ldots,\left(n c_{n}\right)$ applied to the columns, the result is a collection of pairwise orthogonal latin squares $A_{1}, A_{2}, \ldots, A_{t}$ such that cell $(i, j)$ of $A_{m}$ is occupied by $k$ if and only if cell $(i, j)$ of $P_{m}$ is occupied by $k$. That is to say, the pairwise orthogonal partial latin squares $P_{1}, P_{2}, \ldots$, $P_{t}$ are embedded in the pairwise orthogonal latin squares $A_{1}, A_{2}, \ldots, A_{t}$. We have proved the following theorem.

THEOREM. Any collection of pairwise orthogonal finite partial latin squares can be embedded in pairwise orthogonal finite latin squares.

\section{REFERENCES}

1. R. C. Bose and S. S. Shrikhande, On the falsity of Euler's conjecture about the nonexistence of two orthogonal Latin squares of order $4 t+2$, Proc. Nat. Acad. Sci. U.S.A. 45 (1959), 734-737. MR 21 \#3343.

2. Trevor Evans, Embedding incomplete Latin squares, Amer. Math. Monthly 67 (1960), 958-961. MR 23 \# A68.

3. B. Ganter, Endliche Vervollständigung endlicher partieller Steinerscher Systeme, Arch. Math., (Basel) 22 (1971), 328-332. MR 45 \#3218.

4. - Partial pairwise balanced designs (to appear).

5. R. W. Quackenbush, Near vector spaces over $G F(q)$ and $(v, q+1,1)-B I B D s$, Linear Algebra Appl. 10 (1975), 259-266.

Centre de Recherches Mathématiques, Université de Montréal, Montréal, Canada

Current address: Department of Mathematics, Auburn University, Auburn, Alabama 36830 